\title{
An Herbal Formula LI85008F Inhibits Lipogenesis in 3T3-L1 Adipocytes
}

\author{
Krishanu Sengupta ${ }^{1}$, Trimurtulu Golakoti ${ }^{2}$, Venkateswara Rao Chirravuri ${ }^{3}$, Ajit Kumar Marasetti ${ }^{1}$ \\ ${ }^{1}$ Cellular and Molecular Biology Division, Laila Impex R \& D Center, Vijayawada, India; ${ }^{2}$ Drug Discovery and Development Divi- \\ sion, Laila Impex R \& D Center, Vijayawada, India; ${ }^{3}$ Phytochemistry Division, Laila Impex R \& D Center, Vijayawada, India. \\ Email: krishanu@lailanutra.com
}

Received July $11^{\text {th }}, 2011$; revised August $5^{\text {th }}, 2011$; accepted September $12^{\text {th }}, 2011$.

\begin{abstract}
The present study demonstrates a novel herbal formulation LI85008F inhibiting adipocyte differentiation and potentiates lipolysis in 3T3-L1 mouse adipocytes. LI85008F is formulated by combining extracts of three Indian herbs Moringa oleifera, Murraya koenigii and Curcuma longa. Oil red $O$ staining of 3T3-L1 adipocytes reveals that LI85008F is a synergistic formulation that inhibits adipocyte differentiation in a dose dependent manner and concurrently down regulates the key adipogenic transcription factors Peroxisome Proliferator-Activated Receptor gamma (PPAR $\gamma)$ and $C C A A T / e n h a n c e r$ binding protein $\alpha(C / E B P)$. LI85008F confers significant reductions in intracellular triglyceride content in a dose dependent manner. Evidence suggests that LI85008F antagonizes PPARythrough Ser 112 phosphorylation via MAPK/ERK activation. Immunoblot analyses reveal that LI85008F treatment also down regulates the protein expressions of key PPARy responsive gene products such as Adipocyte differentiation related protein (ADRP), CD36, Adipocyte specific binding protein $2(a P 2)$ and perilipin. In differentiated adipocytes culture, LI85008F treatment results in significantly $(p=0.0169)$ increased lipolysis as measured by the release of glycerol. LI85008F does not exhibit cytotoxic effect on adipocytes. Taken together, the results suggest that LI85008F inhibits lipogenesis in adipocytes and concurrently antagonizes PPARy and other lipogenic factors and in addition, potentiates triglyceride mobilization from the fat cells or enhances lipolysis.
\end{abstract}

Keywords: Adipocytes, Adipogenesis, Curcuma Longa, LI85008F, Moringa Oleifera, Murraya Koenigii

\section{Introduction}

Development of obesity is characterized by increased number of fat cells in the body and high intracellular lipid content due to enhanced mitogenesis and differentiation of precursor fat cells, which are regulated by genetic, endocrine, metabolic, neurological, pharmacological, environmental, and nutritional factors [1]. Obesity is a major risk factor for a number of disorders, including diabetes, hypertension, and heart disease [2]. The WorldHealth Organization estimates that by 2020 , two-thirds of the global disease burden will be attributable to chronic diseases associated with obesity [3]. Obesity may partially be prevented or controlled by non-pharmaceutical interventions such as employing diet with restricted calorie along with adequate physical exercise and eating habit modification programs or by using pharmaceutical interventions. However, the current choices for anti-obesity interventions are very limited and some anti-obesity medicines have serious or even life-threatening side effects [4]. Therefore, there is an increasing demand for alternative strategies to control obesity, which would be effective and have minimal or no adverse effects.

Adipocytes are the major cellular component in fat tissue; excessive growth, differentiation and hypertrophy of adipocytes are the fundamental processes of obesity. Maturation of adipocytes occurs from the pre-existing adipocyte progenitor cells (preadipocytes) that are prensent in the body [5]. Therefore, from a pathophysiologcal point of view, both the proliferation and differentiation of preadipocytes into mature adipocytes are the critical factors in development of obesity. Therefore, inhibition in adipogenesis is a potential target for treating obesity. Until recently, several cell types have been shown to undergo in vitro lipogenic differentiation into adipocytes. Among them, 3T3-L1 pre-adipocyte is well characterized cellular model for studying adipogenesis process. Upon induction by chemical cocktail, 3T3-L1 preadipocytes differentiate to form mature adipocytes, with accumulation of triglyceride (TG) as one of the hallmarks of adipogenesis [6]. 
The traditional Indian medicinal plants derived products have been reported to be effective for treating various metabolic and systemic disorders [7]. By employing the in vitro cell based adipogenesis model as a screening tool, we selected Moringa oleifera, Murraya koenigii and Curcuma longa extracts, which exhibited adipogenesis inhibition potential. Moringa oleifera is commonly known as the "drumstick" tree [8]. This plant has medicinal importance and is being used in folk medicine for treatment of variety of human ailments such as rheumatism, paralysis and epilepsy [9]. It also has great value as a food ingredient. The leaves, pods, seeds, flowers, and roots of the $M$. oleifera tree are edible and nontoxic [10].

Murraya koenigii is commonly known as curry leaf. This plant is native to India and is widely available throughout the Indian subcontinent excluding the high altitudes of the Himalayas. Curry leaf is used in folk medicine in India and in other Asian countries as analgesic, astringent, antidysenteric, febrifuge, hypolipidemic and hypoglycemic agent $[11,12]$.

Turmeric (Curcuma longa) has a long tradition of being used as a common spice in the Indian subcontinent and by many cultures across the globe. In Chinese and Ayurvedic systems of medicine, it has been described as an antiinflammatory agent, and used for the treatment of jaundice, menstrual disturbances, hematuria, hemorrhage, and colic etc. It also possesses potent anti-oxidant ability. Turmeric extracts are also used as hepatoprotective, nephroprotective, anti-asthmatic, anti-tumor agents; and as a detoxifier and regenerator of liver tissue etc. Curcumins are the group of active compounds in turmeric [13].

In the present study, we describe the anti-obesity efficacy of LI85008F, a novel herbal formulation, containing the extracts of Moringa oleifera, Murraya koenigii and Curcuma longa, which synergistically inhibit the adipogenesis process in 3T3-L1 cells. In addition, this botanical composition also increases the potential to break down the stored fat in mature adipocytes. This study also indicates that the herbal formulation LI-85008F concurrently antagonizes $\operatorname{PPAR} \gamma$ and down regula- tes the key adipogenic factors responsible for lipogenesis process.

\section{Materials and Methods}

\subsection{Chemicals and Reagents}

Isopropyl alcohol, Insulin, dexamethasone, 3-isobutyl1-me-thylxanthine (IBMX), Oil red O, Dulbecco's modified Eagle's medium (DMEM) and anti-actin antibody were obtained from Sigma Chemical Co. (St. Louis, MO). Fetal bovine serum was purchased from Hyclone (Logan, UT). Anti-CCAAT/enhancer binding protein $\alpha(\mathrm{C} / \mathrm{EBP} \alpha)$, Anti-Peroxisome Proliferator-Activated Receptor (PPAR) $\gamma 2 / 1$ and anti-Phospho PPAR $\gamma$ (Ser112) antibodies were procured from Millipore (Billerica, MA). Antibodies specific to perilipin A was purchased from Abcam (Cambridge, UK); Anti-ADRP (H-80), Anti-CD36 and Adipocyte specific binding protein $2(\mathrm{aP} 2)$ antibodies were purchased from R\&D Systems Inc. (Minneapolis, MN). Antibodies specific to ERK1/2, phospho-ERK1/2 antibodies and MAPK/ERK kinase (MEK) specific inhibitor U0126 were purchased from Cell Signaling Technology (Beverly, MA).

\subsection{Plant Materials}

Fresh plant raw materials were collected from local areas in Andhra Pradesh, India. Their voucher specimens are preserved in the Taxonomy Division at Laila Impex R\&D Centre, Vijayawada, India.

Moringa olefera. Approximately one kilogram of dried leaves of Moringa olefera ( $M$. olifera) was pulverized to coarse powder, extracted with alcohol at reflux for $1 \mathrm{hr}$. Extraction process was repeated three times using alcohol. All the extracts were combined and were evaporated under reduced pressure. The final yield was $100 \mathrm{~g}$.

Murrya koenigii. Approximately one kilogram of shade dried leaves of $M$. koenigii was pulverized to coarse powder, and extracted three times with $60 \%$ aqueous alcohol at $70^{\circ} \mathrm{C}$ for $2 \mathrm{~h}$. The combined hydro-alcohol extracts were evaporated in vacuum. The final yield was $80 \mathrm{~g}$.

Curcuma longa. Approximately one kilogram of shade dried rhizomes of Curcuma longa (C. longa) was pulverized to coarse powder, and extracted with 6 liters of alcohol at reflux for $1 \mathrm{hr}$. The extraction process was repeated for three times. The alcohol extract of $C$. longa was subjected to column chromatography over silica gel using Dichloromethane/methanol mixture. The fraction containing curcuminoids were combined, evaporated and the residue crystallized from ethanol/water mixture to obtain enriched extract containing $95 \%$ total curcuminoids.

\subsection{Cell Culture}

3T3-L1 mouse embryo fibroblasts were obtained from American Type Culture Collection (Manassas, VA) and cultivated in maintenance medium comprised of DMEM supplemented with $10 \%$ fetal bovine serum (FBS) 100 $\mathrm{U} / \mathrm{ml}$ penicillin, $100 \mu \mathrm{g} / \mathrm{ml}$ streptomycin, $1 \mathrm{mM}$ sodium pyruvate and $4.5 \mathrm{~g} / \mathrm{L}$ D-glucose. The dry powdered plant extracts and the formulations were dissolved in DMSO and the final concentration of DMSO in the culture was $0.1 \%(\mathrm{v} / \mathrm{v})$ in all experiments.

\subsection{Adipogenesis Assay}

Equal number of 3T3-L1 cells $(60,000$ cells per well) was seeded in each well of 24-well tissue culture plates and grown to confluence. Cells were pretreated with different concentrations of extracts of either $M$. olifera or $M$. koenigii or C. longa or different combinations of these 
extracts for $2 \mathrm{~h}$ and incubated with the differenttiation medium (maintenance medium supplemented with $500 \mathrm{nM}$ insulin, $1 \mu \mathrm{M}$ dexamethasone, and $0.5 \mathrm{mM} 3$-isobutyl1-methylxanthine) for 2 days. Thereafter, the cells were further maintained in the post-differentiation medium comprised of the maintenance medium and $100 \mathrm{nM}$ of insulin in presence or absence of individual plant extracts and their combinations for 8 days. The control cultures received only $0.1 \%$ (v/v) DMSO as the vehicle.

The intracellular lipid accumulation was measured by staining the cells with Oil Red O. Briefly, the $10 \%$ formaldehyde-fixed cells were washed with $60 \%$ Isopropyl alcohol and the air dried cells were stained with Oil red O for $10 \mathrm{~min}$ at room temperature. Unbound stain was washed with distilled water and the fat accumulation was examined under a Nikon Eclipse TS 100 microscope (400 $\times$ ) equipped with a Nikon Coolpix camera (Nikon Corporation, Japan). The bound dye was eluted with $100 \%$ isopropyl alcohol, and the optical density (OD) was read at $550 \mathrm{~nm}$ using a microplate reader (Bio-Rad, USA). Percent inhibition of adipogenesis/lipogenesis in test samples treated culture wells was calculated in comparison with the $\mathrm{OD}$ in vehicle control treated cultures.

\subsection{Cellular Triglyceride Assay}

Preadipocytes were treated with different concentrations of LI85008F during differentiation as described in adipogenesis assay procedure. Intracellular triglyceride contents were measured quantitatively with Adipogenesis Assay Kit (BioVision, Mountain View, CA). Briefly, the culture wells containing adipocytes were washed with Phosphate Buffered Saline (PBS) and the intracellular lipid was extracted in Lipid extraction solution at $90^{\circ} \mathrm{C}-100^{\circ} \mathrm{C}$ for $30 \mathrm{~min}$. Fifty micro liters of cellular extracts containing lipids were transferred to each well of a 96 well assay plate and $21 \mu \mathrm{l}$ of lipase was added to each well. Thereafter, equal volume of triglyceride reaction mixture was added to each well and incubated at $37^{\circ} \mathrm{C}$ for $30 \mathrm{~min}$. In parallel, triglyceride standard was run in the same assay plate at different concentrations ranging from 0 to $10 \mathrm{nM}$. Finally, the absorbance was read at $570 \mathrm{~nm}$ using a microplate reader (Bio-Rad, USA). Background colors were corrected by subtracting the value derived from 0 triglyceride standard from all readings. Protein contents in each well were determined by BCA method (BioRad Laboratories, Hercules, CA). Intracellular triglyceride contents were estimated quantitatively using a standard curve and the triglyceride values were normalized to $\mathrm{nM}$ per $\mathrm{mg}$ of protein.

\subsection{Lipolysis Assay}

The lipolysis efficacy of LI85008F was evaluated by measuring the released glycerol in the 3T3-L1 culture supernatants. Briefly, equal number of 3T3-L1 preadipocytes was differentiated into mature adipocytes. In every culture well, 90\% - 95\% cells became differentiated, containing numerous intracellular vesicles visible under microscope. Thereafter, the wells were washed two times with pre-warmed Hank's Balanced Salt Solution (HBSS). Washed cells were treated with $50 \mu \mathrm{g} / \mathrm{ml}$ of LI85008F mixed in phenol red free DMEM supplemented with $2 \%$ Bovine serum albumin (BSA) for $4 \mathrm{~h}$. Cell culture supernatants were collected and clarified at 10,000 $\mathrm{g}$ for $5 \mathrm{~min}$ at $4^{\circ} \mathrm{C}$. Released glycerol content in the culture supernatants was measured with glycerol reagent according to the protocol provided in Adipolysis Assay Kit (Millipore, Billerica, MA).

\subsection{Immunoblot Analysis}

Briefly, the treated cells were washed twice with chilled PBS and the cell lysates were prepared in a lysis buffer (50 mM Tris, $\mathrm{pH} 7.5,150 \mathrm{mM}$ sodium chloride, $2 \mathrm{mM}$ EDTA, 1\% Triton X-100, 0.5\% sodium deoxycholate, $1 \mathrm{mM}$ sodium vanadate, $1 \mathrm{mM}$ sodium fluoride, $1 \mu \mathrm{g} / \mathrm{mL}$ aprotinin, $1 \mu \mathrm{g} / \mathrm{mL}$ leupeptin, $1 \mu \mathrm{g} / \mathrm{mL}$ pepstatin and 1 $\mathrm{mM}$ phenylmethylsulfonyl fluoride). The cell lysates were clarified at $14,000 \mathrm{~g}$ for $20 \mathrm{~min}$ at $4^{\circ} \mathrm{C}$. The protein concentrations were estimated by Bradford reagent.

Equal amount of proteins was resolved in SDS-PAGE. Following SDS-PAGE, the electro-blotted nitrocellulose membranes were reacted with relevant primary antibody specific to $\mathrm{C} / \mathrm{EBP} \alpha$, PPAR $\gamma$, Phospho-PPAR $\gamma$ (Ser112), ADRP, CD36, perilipin A, ERK1/2 and phospho-ERK1/2. The same membrane was reprobed with anti-actin antibody. Specific signals were detected with enhanced chemiluminescence (Thermo scientific, USA) and the signal intensities were analyzed using Molecular Imaging Software, version 4.0 (Eastman Kodak Company, Rochester, NY).

\subsection{Cell Proliferation Assay}

MTT [3-(4,5-dimethylthiazol-2-yl)-2,5-diphenyltetrazolium bromide] incorporation based cell proliferation assay was performed by MTT cell proliferation assay kit (Roche Applied Sciences, Germany) following the procedure described earlier [14] with some modifications. Briefly, equal number of 3T3-L1 pre-adipocytes (5000 cells/well) was plated in 96-well flat-bottom plates and differentiated as above. Matured adipocytes were incubated with LI85008F at various concentrations for a period of $48 \mathrm{~h}$ hours. Vehicle control culture wells received only a maximum of $0.1 \%$ DMSO. Thereafter, $0.5 \mathrm{mg} / \mathrm{ml}$ of MTT reagent was added to each well and the microplate was incubated further for $4 \mathrm{~h}$ at $37^{\circ} \mathrm{C}$ in presence of $5 \% \mathrm{CO}_{2}$. Finally, the cells were solubilized and the absorbance was read at $540 \mathrm{~nm}$ in a microplate reader (BioRad, USA). The cell proliferation index of the LI85008F treated wells was calculated from the mean absorbance (from quadruplicate wells), 
considering that of the vehicle treated cultures as $100 \%$.

\subsection{Lactate Dehydrogenase Assay}

Cytotoxic potential of LI85008F was evaluated by measuring the lactate dehydrogenase (LDH) leaked into the 3T3-L1 Adipocyte culture supernatant following instructtions provided by the vendor (LDH Cytotoxicity Detection Kit ${ }^{\text {Plus }}$, Roche Applied Sciences, Germany). The leaked LDH is directly proportional to the cell damage done by any cytotoxic compound. Briefly, equal number of 3T3-L1 adipocytes was treated with LI85008F at various concentrations and incubated for $48 \mathrm{~h}$. Vehicle control culture wells received only a maximum of $0.1 \%$ DMSO. The cell free culture supernatants were mixed with catalyst and dye solution and allowed to incubate for $15 \mathrm{~min}$ at room temperature. Finally, the color reaction was stopped and the absorbance was measured at $492 \mathrm{~nm}$ in a microplate reader (BioRad, USA).

\subsection{Statistical Analysis}

Results are expressed as mean \pm standard deviation. The significance of data was evaluated using Student's $t$-test for comparison between two groups. The values of $\mathrm{p}<$ 0.05 were considered statistically significant.

\section{Results}

\subsection{LI85008F Synergistically Inhibits Lipid Accumulation in 3T3-L1 Adipocytes}

Based on the standard protocol [15], we screened the aqueous, aqueous-ethanol and ethanol extracts of two hundred Indian medicinal plants mentioned in the Ayurveda for various medicinal uses. Inhibition in lipid accumulation was evaluated in Oil Red O stained 3T3-L1 adipocytes and best three herbal extracts were selected viz., Moringa olefera, Murrya koenigii and Curcuma longa. Table 1 shows alcohol extract of $M$. olefera, aqueous alcohol extract of $M$. koenigii and $10 \mu \mathrm{g} / \mathrm{ml}$ of $95 \%$ curcumanoids extract of C. longa inhibit $25.70 \%, 24.86 \%$ and $29.50 \%$ lipid accumulation in mature adipocytes, respectively.

We combined the extracts of $M$. olefera, $M$. koenigii and C. longa in different ratios. Interestingly, we found that out of fourteen compositions, only LI85008F, a composition containing 6:3:1 ratio of $M$. olefera, $M$. koenigii and $C$. longa extracts, respectively exhibited $40.5 \%$ inhibition in lipid accumulation in adipocytes, which is synergistically better than the activities of the individual extracts (Table 1). Figures 1(a)-(d) show Oil Red O staining in 3T3-L1 adipocytes differentiated in presence or absence of 10, 25 and $50 \mu \mathrm{g} / \mathrm{ml}$ of LI85008F. Inhibition of adipocytes differentiation was accompanied by a significant reduction in cezllular triglyceride levels in LI85008F treated cells. Quantitative analyses show that 10,25 and $50 \mu \mathrm{g} / \mathrm{ml}$ of LI85008F provides $23.94 \%(p=0.0031), 51.83 \%(p<0.0001)$ and $58.29 \%(\mathrm{p}<0.0001)$ reductions in intracellular triglyceride content, respectively, as compared to vehicle treated adipocytes (Figure 1(e)). Together, these observations strongly suggest that LI850085 inhibits lipogenesis in 3T3-L1 adipocytes.

Table 1. Herbal extracts inhibit adipogenesis in 3T3-L1 cells.

\begin{tabular}{ccc}
\hline Treatments & $\begin{array}{c}\text { Treatment } \\
\text { Concentration }\end{array}$ & \% inhibition of adipogenesis \\
\hline Moringa olefera leaf extract & $10 \mu \mathrm{g} / \mathrm{ml}$ & 25.70 \\
& $25 \mu \mathrm{g} / \mathrm{ml}$ & 28.56 \\
& $50 \mu \mathrm{g} / \mathrm{ml}$ & 31.32 \\
Murraya koenigii leaf extract & $10 \mu \mathrm{g} / \mathrm{ml}$ & 24.86 \\
& $25 \mu \mathrm{g} / \mathrm{ml}$ & 26.72 \\
Curcuma longa extract & $50 \mu \mathrm{g} / \mathrm{ml}$ & 30.97 \\
& $10 \mu \mathrm{g} / \mathrm{ml}$ & 29.50 \\
& $25 \mu \mathrm{g} / \mathrm{ml}$ & 36.36 \\
& $50 \mu \mathrm{g} / \mathrm{ml}$ & 39.05 \\
\hline
\end{tabular}



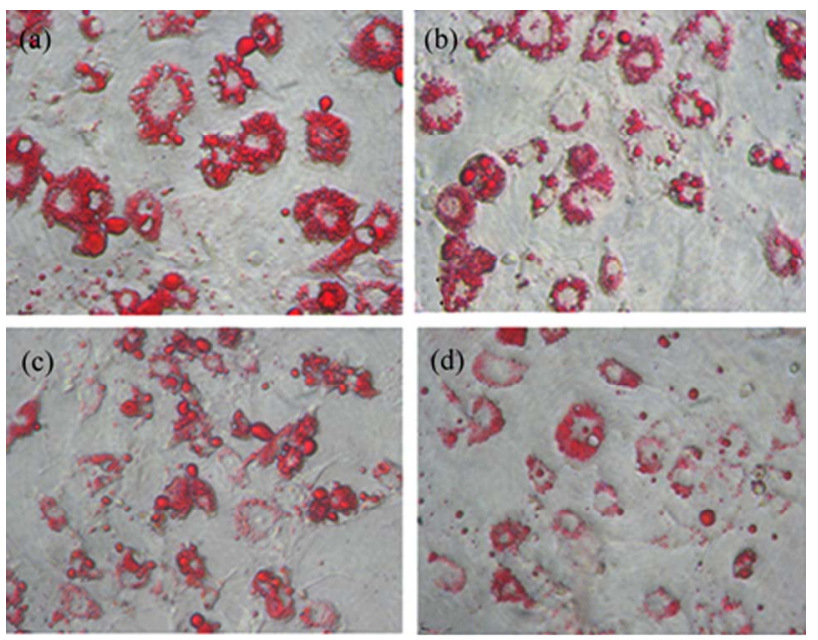

(e)

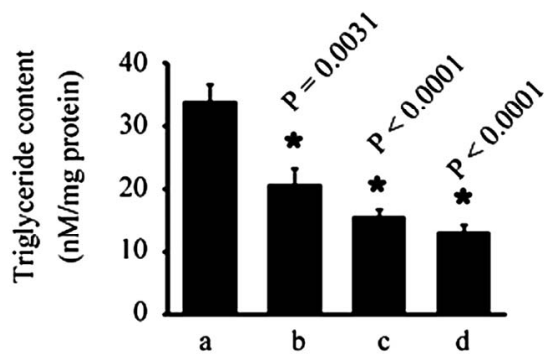

Figure 1. LI85008F suppresses lipid accumulation in 3T3$\mathrm{L} 1$ cells. 3T3-L1 cells were induced to differentiate with adipogenic cocktail containing $500 \mathrm{nM}$ insulin, $1 \mu \mathrm{M}$ dexamethasone, and $0.5 \mathrm{mM}$ 3-isobutyl-1-methylxanthine in presence or absence different concentrations of LI85008F as described in Methods and Procedures. Lipids were stained with Oil Red O. Representative photomicrographs show lipid accumulation in the cells treated with $0.1 \%$ DMSO as vehicle control (a); cells treated with 10,25 and $50 \mu \mathrm{g} / \mathrm{ml}$ of LI85008F ((b), (c), and (d), respectively). (e), Bar diagram represents mean \pm SD of intracellular triglyceride content in differentiating adipocytes concomitantly treated with either vehicle (a) or 10, 25 and $50 \mu \mathrm{g} / \mathrm{ml}$ of LI85008F ((b), (c) and (d), respectively). $n=5, p<0.05$ vs. vehicle control considered as significant (paired $t$-test).

\subsection{LI85008F Promotes Breakdown of Stored Fat in 3T3-L1 Adipocytes}

The effect of LI85008F on lipid break down or lipolysis in matured 3T3-L1 adipocytes was determined by measuring released glycerol into the culture medium. LI85$00-8 \mathrm{~F}$ treatment $(50 \mu \mathrm{g} / \mathrm{ml})$ significantly $(\mathrm{p}=0.0169)$ enhanced lipolysis or fat breakdown in matured 3T3-L1 adipocytes. Vehicle treated control $(0.1 \%$ DMSO $)$ cultures $(\mathrm{n}=6)$ released $2.626 \pm 0.339 \mu \mathrm{g} / \mathrm{ml}$ of glycerol in 4 $\mathrm{h}$, whereas, $50 \mu \mathrm{g} / \mathrm{ml}$ of LI85008F treated cultures $(\mathrm{n}=6)$ released $3.364 \pm 0.294 \mu \mathrm{g} / \mathrm{ml}$ (Figure 2). Thus, $50 \mu \mathrm{g} / \mathrm{ml}$ of LI85008F provides $27.96 \%$ increase in lipolysis in com- parison with the vehicle treated 3T3-L1 adipocytes.

\subsection{LI85008F Down Regulates Adipogenic Modulators in 3T3-L1 Adipocytes}

3T3-L1 adipogenic differentiation requires synergistic activity of multiple transcription factors and adipogenic modulatory factors including PPAR $\gamma, \mathrm{C} / \mathrm{EBP}, \mathrm{aP2}$ etc. [16]. Among them, PPAR $\gamma$ is the key transcription factor which is up-regulated and activated during the adipogenesis process and it further up-regulates several downstream adipogenic modulators such as Adipocyte differentiation related protein (ADRP), CD36, perilipin etc. The immunoblot experiments show that LI85008F treatment down regulates C/EBP and PPAR $\gamma$ protein expressions in 3T3-L1 adipocytes. Similar effects are also observed in the expressions of PPAR $\gamma$ responsive adipogenic modulators such as ADRP, CD36, aP2, and perilipin (Figure 3). Formation of lipid droplets in adipocytes as well as lipid breakdown or lipolysis is regulated by a mem- brane protein surrounding the lipid droplets, perilipin, which is a crucial downstream target of PPAR $\gamma$ for adipocytes differentiation.

\subsection{LI85008F Deactivates the PPAR $y$ in 3T3-L1 Adipocytes}

PPAR $\gamma$ phosphorylation at Ser112 via activated ERK/ MAPK pathway is crucial for deactivation of PPAR $\gamma$ [17-19]. Immunoblot assays reveal that LI85008F treatment decreased PPAR $\gamma$ expression in adipocytes and concurrently increases the phosphorylation of PPAR $\gamma$ at Ser112 (Figure 4(a)). LI85008F also activates the MAPK pathway through increased ERK phosphorylation in adipopcytes (Figure 4(b)). The peak level of ERK phosphorylation reached

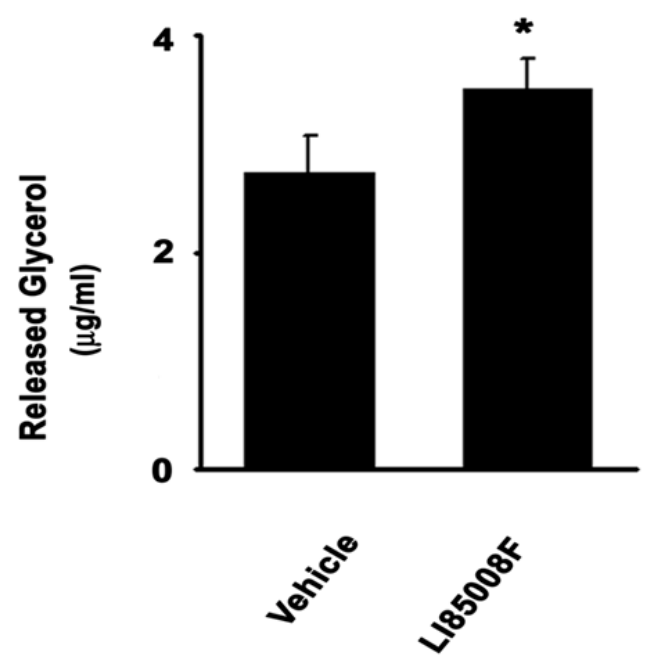

Figure 2. LI85008F increases lipolysis. Differentiated 3T3-L1 mature adipocytes were incubated with $50 \mu \mathrm{g} / \mathrm{ml}$ of LI85008F for $4 \mathrm{~h}$. Released glycerol was measured quantitatively with Adipolysis assay kit as described in Materials and Methods. The bars represent mean $\pm S D . N=6 .{ }^{*} p=0.0169$, vs. vehicle. 


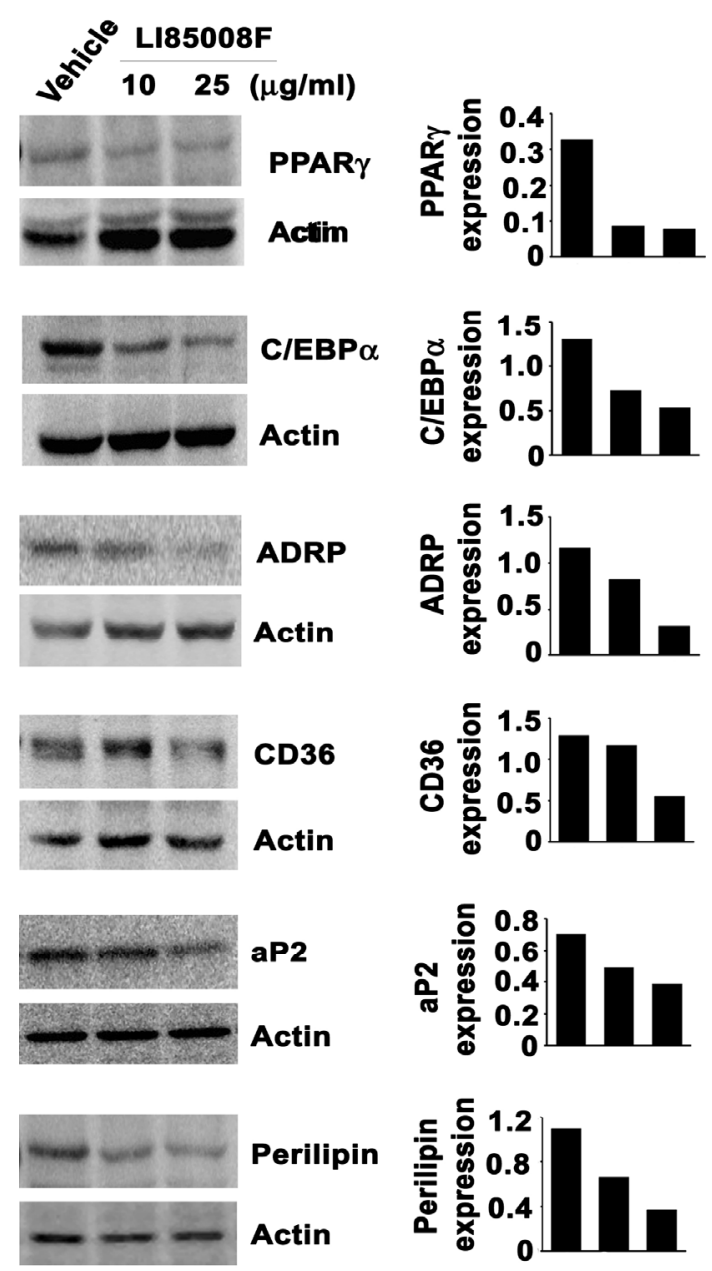

Figure 3. LI85008F down regulates the expressions of adipogenic transcription factors and related adipogenic modulators of 3T3-L1 differentiation. Representative immunoblots depict down-regulation of PPAR $\gamma, \mathrm{C} / \mathrm{EBP} \alpha$, ADRP, CD36, aP2 and perilipin as indicated. 3T3-L1 mouse preadipocytes were differentiated in absence or presence of $10 \mu \mathrm{g} / \mathrm{ml}$ or 25 $\mu \mathrm{g} / \mathrm{ml}$ of LI85008F as described in Materials and Methods. Vehicle control cultures received $0.1 \%$ of DMSO. Expression of actin protein was evaluated in each blot as the internal control. Expression of each protein was measured densitometrically and normalized with actin expression. Bar diagrams show the normalized expression of each protein in arbitrary units.

after $30 \mathrm{~min}$ of LI85008F treatment. Next, to explore whether LI85008F treatment increases PPAR $\gamma$ phosphorylation via MAPK/ERK activation, we pretreated the adipocytes with U0126, a MAPK/ERK kinase (MEK) specific inhibitor. Interestingly, we observed that LI85008F could not increase PPAR $\gamma$ phosphorylation in U0126-pretreated cells; whereas, LI85008F increased PPAR $\gamma$ phosphorylation by 2.71 fold in comparison with the vehicle treated cells. U0126 treated cells showed 3.67-fold increase in PPAR $\gamma$ phosphorylation above the baseline (Figure 4(c)).

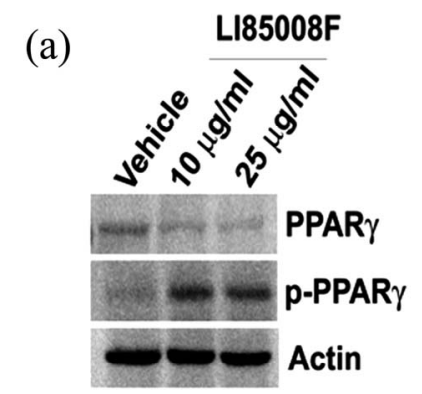

(b)

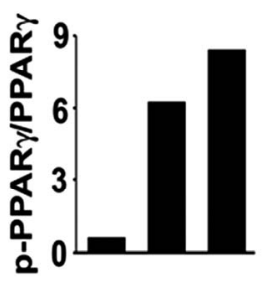

(c)
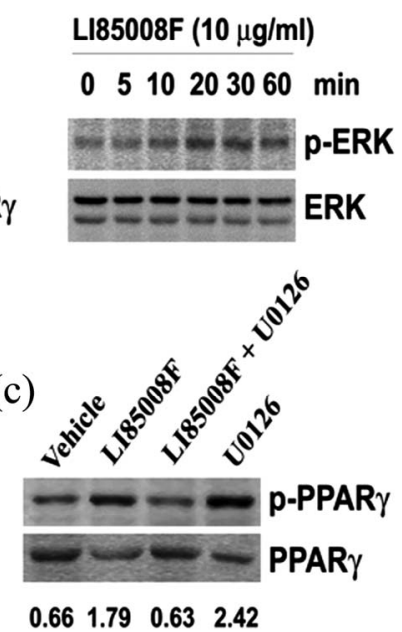

Figure. 4 LI85008F phosphorylates PPAR $\gamma$ through ERK/MAPK activation. (a) 3T3-L1 preadipocytes were incubated with adipogenic cocktail in presence of either LI85008F (10 or $25 \mu \mathrm{g} / \mathrm{ml}$ ) or vehicle (0.1\% DMSO) for $4 \mathrm{~h}$. Representative immunoblots show PPAR $\gamma$ and phospho-PPAR $\gamma$ (Ser112) expressions in cell lysates. In bar diagram, each bar shows the ratio of p-PPAR $\gamma$ and PPAR $\gamma$ in arbitrary units. (b) 3T3-L1 preadipocytes were treated with $10 \mu \mathrm{g} / \mathrm{ml}$ of LI85008F for different periods as indicated. Immunoblots show p-ERK and ERK protein expressions in respective cell lysates. (c) 3T3-L1 preadipocytes were pretreated with either $10 \mu$ M U0126 or vehicle $(0.1 \%$ DMSO) for $30 \mathrm{~min}$ and further incubated for $4 \mathrm{~h}$ with or without $10 \mu \mathrm{g} / \mathrm{ml}$ of LI85008F. Representative immunoblots show the p-PPAR $\gamma$ and PPAR $\gamma$ protein expressions in respective cell lysates as indicated. The values (in arbitrary units) indicate the ratios between densitometric units of p-PPAR $\gamma$ and PPAR $\gamma$ expressions, respectively.

\subsection{LI85008F does Not Effect Proliferation of Adipocytes}

To determine whether LI85008F inhibits the proliferation of preadipocytes, we treated mature 3T3-L1 adipocytes in the presence and absence of varying doses of LI85008F for $48 \mathrm{~h}$, thereafter, we measured cell proliferation. Figure 5(a) shows addition of up to $100 \mu \mathrm{g} / \mathrm{ml}$ of LI85008F does not affect significantly the proliferation of 3T3-L1 adipocytes. After $48 \mathrm{~h}$ of treatment, $100 \mu \mathrm{g} / \mathrm{ml}$ of LI85008F reduced 3T3-L1 adipocytes proliferation by only $4.26 \%$. To determine whether LI85008F has any cytotoxic effects on adipocytes, we measured released LDH into the culture medium in response to varying doses of LI85008F. Figure 5(b) shows up to $100 \mu \mathrm{g} / \mathrm{ml}$ of LI85008F does not increase LDH release into the 3T3-L1 culture medium. It indicates that LI85008F treatment does not produce any cytotoxic effect on mature adipocytes.

\section{Discussion}

Adipocyte differentiation is critical for the onset and 
(a)

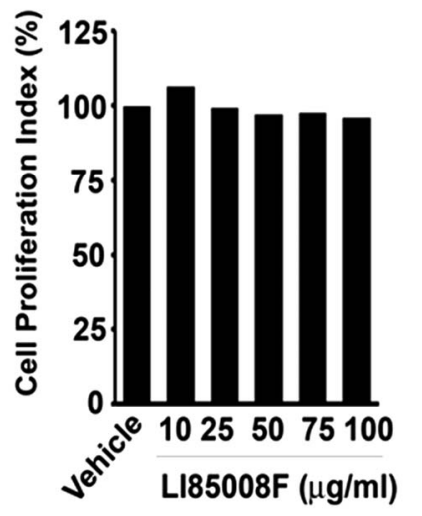

(b)

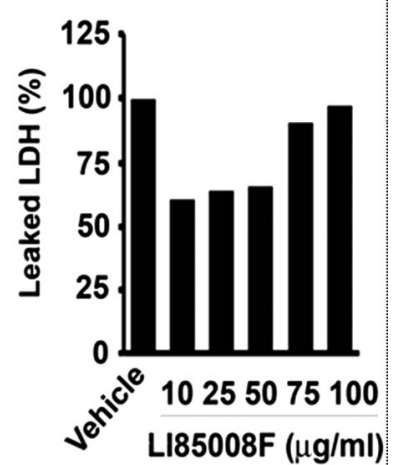

Figure 5. LI85008F does not affect 3T3-L1 adipocyte viability. Equal number of mature 3T3-L1 adipocytes was incubated with LI85008F at various concentrations for $48 \mathrm{~h}$. (a) Cell viability was determined by the MTT incorporation based cell proliferation assay. Assays were performed in quadruplicates for each treatment. Vehicle treated cultures received $0.1 \%$ DMSO. Each bar indicates the cell proliferation index of respective treatments as described in Methods and Procedures. (b) Cytotoxic potential of LI85008F was evaluated by measuring LDH leaked into the adipocyte culture medium. Each bar represents the percentage of secreted LDH in LI85008F treated $(n=4)$ cultures with respect to vehicle treated cultures as indicated.

progression of obesity and other metabolic diseases. The adipogenesis process includes alteration of cell shape, growth arrest and clonal expansion, which leads to a complex sequence of changes in gene expression and lipid storage [18]. Differentiation of fibroblastic preadipocytes into mature adipocytes involves differential regulation of adipogenic genes as well as lipid accumulation. Increase in fat mass is a result of; increase in adipocyte number due to proliferation, and induction of differentiation that stimulates mitotic clonal expansion and irreversible commitment to differentiation [19]. Therefore, the strategies for fat reduction during weight loss intervention may involve either inhibition of lipid accumulation by decreasing adipogenic process and, and/or increased lipid breakdown (lipolysis) and/or induction of programmed cell death or apoptosis. In agreement with these ideas, we intended to search some Indian medicinal herbs which exhibited potential inhibitory effect on adipocyte differentiation. We screened aqueous, aqueous alcohol and ethanol extracts of more than two hundred Indian medicinal plants indicated for treatment of various ailments practiced in Ayurveda, the system of traditional medicine in India.

Previously, extracts from some plants, such as Pinus pinaster, maritime pine tree [20], Ginkgo biloba [20], grape seed extract [21] and green tea [23], have been reported to inhibit adipogenesis in adipocytes. In our study, the screening assays designed in 3T3-L1 mouse preadipocytes reveal that alcohol extract of $M$. olefera, aqueous alcohol extract of $M$. koenigii and enriched extract of $C$. longa inhibit adipogenesis during matu ration of adipocytes (Table 1). Further, we were interested to see whether these extracts can generate a synergistic composition to inhibit the adipogenesis more efficiently than the individual extracts. We tested fourteen compositions prepared from three herbal extracts in preadipocytes differentiation model system. Interestingly, LI85008F, a composition containing 6:3:1 ratio of $M$. olefera, $M$. koenigii and C. longa extracts synergistically inhibited adipogenesis in differentiating 3T3-L1 cells in a dose dependent manner (Table 1). Intracellular lipid staining and triglyceride assay confirm the potential of anti-obesity efficacy of LI85008F (Figure 1). In addition, our study also shows that LI85008F significantly increases glycerol release from mature adipocytes into the culture medium (Figure 2). This observation strongly indicates that LI85008F enhances lipolysis or fat mobilization from the mature fat cells.

During adipogenesis, the adipocyte differentiation and concurrent intracellular lipid accumulation are regulated by adipogenic signals to induce expression of transcripttional activators, mainly of PPAR $\gamma$ and C/EBP families $[23,24]$. These two nuclear factors regulate the complex program of adipogenic gene expression during terminal preadipocytes differentiation in a synchronized manner via triggering expression of multiple adipogenic gene products, such as ADRP, aP2, CD36, perilipin that synergistically lead to adipocyte phenotype $[25,26]$. In the present study, our observations indicate that LI85008F has no effect on growth arrest or cell death (Figure 5) but inhibits differentiation of preadipocytes and concurrently decreasing the expression of nuclear factors $\operatorname{PPAR} \gamma, \mathrm{C} / \mathrm{EBP} \alpha$, and thereby suppressing lipogenesis (Figure 3). These observations provide support in favor of antiadipogenic activity of LI85008F, which is nontoxic to he cells. Previously, we demonstrated a broad spectrum safety of LI85008F in animal models [27]. However, the immunoblot analyses reveal that LI85008F treatment remarkably down regulates the expressions of PPAR $\gamma$ responsive gene products such as ADRP, CD36, aP2 and perilipin (Figure 3). These observations confirm the anti-adipogenic activity of LI85008F through PPAR $\gamma$ antagonism. In addition, these observations also reveal that LI85008F treatment results in (a) decrease in fat store; (b) decrease in availability of fatty acid transport system; (c) provides more susceptibility to hormone sensitive lipase for lipolytic breakdown of stored fat. However, in addition to the adipocyte dedifferentiation activeity of LI85008F, this novel herbal formula also exhibits lypolytic activity in mature adipocytes (Figure 2). At present, the basis of its enhanced lipolytic activity is unexplored. We assume that LI85008F might enhance the lipid mobilization through activation of 3 
adrenergic recaptor [28], as LI85008F strongly induces 3AR protein expression in adipocytes (unpublished observation, data not shown).

Adipocyte differentiation is inhibited when PPAR $\gamma$ is phosphorylated at Ser112 via ERK/MAPK activation, suggesting a mechanism which negatively regulates to limit adipocyte differentiation and lipid accumulation [17-19]. Interestingly, immunoblot data reveal that LI85008F treatment up regulates the ERK/MAPK activation and concurrent phosphorylation of PPAR $\gamma$ at Ser112 in adipocytes, whereas, in presence of U0126, LI85008F fails to phosphorylate PPAR $\gamma$. Therefore, in accordance with the earlier observations, we suggest that LI85008F deactivates $\operatorname{PPAR} \gamma$ via its phosphorylation through MAPK activation and consequently inhibits adipocyte differentiation process.

\section{Conclusions}

Present study demonstrates that LI85008F inhibits lipogenesis in 3T3-L1 cells by reducing preadipocyte differentiation, promotes lipid break down in mature adipocytes without affecting cell proliferation. Evaluation of the anti-obesity efficacy of LI85008F in a suitable animal model is warranted. However, we propose that this non-toxic, novel herbal formula could be a safe and potential candidate for weight management in obese humans.

\section{Acknowledgements}

We sincerely thank Sri G. Ganga Raju, Chairman; Mr. G Rama Raju, Director; and Mr. B. Kiran, CEO of Laila Group of Industries, India for generous support and encouragements. This study was supported by Laila Nutraceuticals, Vijayawada, India.

\section{Declaration of Interests}

The authors declare that there are no competing interests.

\section{REFERENCES}

[1] S. R. Farmer and J. Auwerx, "Adipose Tissue: New Therapeutic Targets from Molecular and Genetic Studies: IASO Stock Conference 2003 Report," Obesity Research, Vol. 5, No. 4, 2004, pp. 189-196. doi:10.1111/j.1467-789X.2004.00157.x

[2] W. J. Lee, E. H. Koh, J. C. Won, M. S. Kim, J. Y. Park and K. U. Lee, "Obesity: The Role of Hypothalamic AMPactivated Protein Kinase in Body Weight Regulation," The International Journal of Biochemistry \& Cell Biology, Vol. 37, No. 11, 2005, pp. 2254-2259. doi:10.1016/j.biocel.2005.06.019

[3] M. Chopra, S. Galbraith and I. Darnton-Hill, "A Global Response to a Global Problem: The Epidemic of over Nutrition," Bulletin of the World Health Organization, Vol. 80, No. 12, 2002, pp. 952-958.

[4] D. Rucker, R. Padwal, S. K. Li, C. Curioni and D. C. Lau,
"Long Term Pharmacotherapy for Obesity and Overweight: Updated Meta Analysis," BMJ, Vol. 335, No. 7631, 2007, pp. 1194-1199.

[5] F. M. Gregore, C. M. Smas and H. S. Sul, "Understanding Adipocyte Differentiation," Physiological Reviews, Vol. 78, No. 3, 1998, pp. 783-809.

[6] J. M. Ntambi and K. Young-Cheul, "Adipocyte Differentiation and Gene Expression," Journal of Nutrition, Vol. 130, No. 12, 2000, pp. 3122S-3126S.

[7] S. Dev, "Ancient-Modern Concordance in Ayurvedic Plants: Some Examples," Environmental Health Perspectives, Vol. 107, 1999, pp. 783-789.

doi:10.1289/ehp.99107783

[8] B. N. Sastri, "The Wealth of India-Raw Materials," CSIR, New Delhi, 1962.

[9] K. M. Nadkarni and A. K. Nadkarni, "The Indian Materia Medica," Popular Prakasan, Bombay, 1976.

[10] J. Dolly, K. R. Prashant, K. Amit, M. Shikha and W. Geeta, "Effect of Moringa oleifera Lam. Leaves Aqueous Extract Therapy on Hyperglycemic Rats," Journal of Ethnopharmacology, Vol. 123, No. 3, 2009, pp. 392-396. doi:10.1016/j.jep.2009.03.036

[11] P. Arulselvan and S. P. Subramanian, "Beneficial Effects of Murraya koenigii Leaves on Antioxidant Defense System and Ultra Structural Changes of Pancreatic BetaCells in Experimental Diabetes in Rats," Chemico-Biological Interactions, Vol. 165, No. 2, 2007, pp. 155-164. doi:10.1016/i.cbi.2006.10.014

[12] S. Yadav, V. Vats, Y. Dhunnoo and J. K. Grover, "Hypoglycemic and Anti-Hyperglycemic Activity of Murraya koenigii Leaves in Diabetic Rats," Journal of Ethnopharmacology, Vol. 82, No. 2-3, 2002, pp. 111-116. doi:10.1016/S0378-8741(02)00167-8

[13] I. Chattopadhyay, K. Biswas, U. Bandyopadhyay and R. K. Banerjee, "Turmeric and Curcumin: Biological Actions and Medicinal Applications," Current Science, Vol. 87, No. 1, 2004, pp. 44-53.

[14] K. Sengupta, J. N. Kolla, A. V. Krishnaraju, N. Yalamanchili, C. V. Rao, T. Golakoti, S. Raychaudhuri and S. P. Raychaudhuri, "Cellular and Molecular Mechanisms of Anti-Inflammatory Effect of Aflapin: A Novel Boswellia serrata Extract," Molecular and Cellular Biochemistry, Vol. 354, No. 1-2, 2011, pp. 189-197. doi:10.1007/s11010-011-0818-1

[15] H. Erding, K. J. Bum, S. Pasha and B. M. Spiegelman, "Inhibition of Adipogenesis through MAP Kinase-Mediated Phosphorylation of PPAR $\gamma$," Science, Vol. 27, Vol. 4, 1996, pp. 2100-2103.

[16] H. S. Camp, A. Chaudhury and T. Leff, “A Novel Potent Antagonist of Peroxisome Proliferator-Activated Receptor Blocks Adipocyte Differentiation but Does Not Revert the Phenotype of Terminally Differentiated Adipocytes," Endocrinology, Vol. 142, No. 7, 2001, pp. 3207-3213. doi:10.1210/en.142.7.3207

[17] Y. Tanabe, M. Koga, M. Saito, Y. Matsunaga and K. Nakayama, "Inhibition of Adipocyte Differentiation by Mechanical Stretching through ERK-Mediated Down- 
regulation of PPAR $\gamma$," Journal of Cell Science, Vol. 117, No. 16, 2004, pp. 3605-3614. doi:10.1242/jcs.01207

[18] F. M. Gregoire, "Adipocyte Differentiation: From FibroBlast to Endocrine Cell," Experimental Biology and Medicine, Vol. 226, No. 11, 2001, pp. 997-1002.

[19] S. Rayalama, M. A. Della-Feraa and C. A. Bailea, "Phytochemicals and Regulation of the Adipocyte Life Cycle," Journal of Nutrition Biochemistry, Vol. 19, No. 11, 2008, pp. 717726. doi:10.1016/i.jnutbio.2007.12.007

[20] M. Dell'Agli and E, Bosisio, "Biflavones of Ginkgo biloba Stimulate Lipolysis in 3T3-L1 Adipocytes," Planta Medica, Vol. 68, No. 1, pp. 76-79. doi:10.1055/s-2002-19876

[21] D. A. Moreno, N. Ilic, A. Poulev, D. L. Brasaemle, S. K. Fried and I. Raskin, "Inhibitory Effects of Grape Seed Extract on Lipases," Nutrition, Vol. 19, No. 10, 2003, pp. 876-879. doi:10.1016/S0899-9007(03)00167-9

[22] M. Mori and N. Hasegawa, "Superoxide Dismutase Activity Enhanced by Green Tea Inhibits Lipid Accumulation in 3T3-L1 Cells," Phytotherapy Research, Vol. 17, No. 5, 2003, pp. 566-567. doi:10.1002/ptr.1182

[23] F. M. Gregoire, C. M. Smas and H. S. Sul, "Understanding Adipocyte Differentiation," Physiological Reviews, Vol. 78, No. 3, 1998, pp. 783-809.
[24] P. Tontonoz, E. Hu and B. M. Spiegelman, "Regulation of Adipocyte Gene Expression and Differentiation by Peroxisome Proliferator Activated Receptor Gamma," Current Opinion in Genetics \& Development, Vol. 5, No. 5, 1995, pp. 571-576. doi:10.1016/0959-437X(95)80025-5

[25] E. D. Rosen and B. M. Spiegelman, "Molecular Regulation of Adipogenesis," Annual Review of Cell and Developmental Biology, Vol. 16, 2000, pp. 145-171. doi:10.1146/annurev.cellbio.16.1.145

[26] M. Kimihiko, J. M. Peters and F. J. Gonzalez, "PPAR $\beta /$ $\delta$ Potentiates PPAR $\gamma$-Stimulated Adipocyte Differentiation," FASEB Journal, Vol. 18, No. 12, 2004, pp. 1477-1479.

[27] A. V. Krishnaraju, D. Sundararaju, P. Srinivas, C. V. Rao, K. Sengupta and G. Trimurtulu, "Safety and Toxicological Evaluation of a Novel Anti-Obesity Formulation LI85008F in Animals," Toxicology Mechanisms and Methods, Vol. 20, No. 2, 2010, pp. 59-68. doi: $10.3109 / 15376510903483722$

[28] K. J. Soeder, S. K. Snedden, W. Cao, G. J. Della-Rocca, K. W. Daniel, L. M. Luttrell and S. Collins, "The 3-Adrenergic Receptor Activates Mitogen-activated Protein Kinase in Adipocytes through a $\mathrm{G}_{\mathrm{i}}$-Dependent Mechanism," The Journal of Biological Chemistry, Vol. 274, No. 17, 1999, pp. 12017-12022. 